Series A

\author{
I. MATHEMATICA
}

498

\title{
NULL ORLICZ CLASSES OF RIEMANN SURFACES
}

BY

ARTHUR E. OBROCK

HELSINK I 1972

S U O M A L A I N E N T I E D E A K A T E M I A

doi:10.5186/aasfm.1971.498 
Copyright (C) 1972 by

Academia Scientiarum Fennica

ISBN $-951-41-0031-\mathrm{X}$

Communicated 14 May 1971 by P. J. MyrberG 


\section{Null Orlicz classes of Riemann surfaces}

1. Introduction. Maurice Heins [1] has used null Hardy classes to classify Riemann surfaces. His success depends to a considerable extent on the Myrberg example, the Szegö-Solomentsev Theorem and the way that $\left|z^{p}\right|$ smears the behavior of $\varphi_{p}(x) \equiv x^{p}$ over an angular sector (for references see [1]). In this paper we attempt to refine this linear classification by using the non-linear lattice-like null Orlicz classes $\mathscr{C}_{\varphi}$. We obtain partial results on the problem of characterizing the inclusion relations between the classes $\mathscr{Q}_{\varphi}$ in terms of the growth relations between the functions $\varphi$.

To be precise we say that a homeomorphism $\varphi(r)$ of $[0, \infty)$ onto itself is an Orlicz function if

$$
\begin{gathered}
\varphi(r) \text { is convex in } \log r, \\
\log r=\mathrm{o}(\varphi(r)) .
\end{gathered}
$$

In other words the Orlicz condition (1) means that $\Phi(x)=\varphi\left(e^{x}\right)$ is convex and the de La Vallée Poussin condition (2) means that $x^{-1} \Phi(x) \rightarrow \infty$ as $x \rightarrow \infty$. In this paper the symbol $" \varphi »$ will be reserved to denote an Orlicz function. Although functions other than $\varphi$ could be considered, the SzegöSolomentsev Theorem applies naturally to these.

If $S$ denotes a Riemann surface let $\mathbf{A}(S), \mathbf{P}(S)$ denote its family of analytic, positive harmonic functions respectively. Let

$$
\mathbf{H}_{\varphi}(S)=\{f \in \mathbf{A}(S): \varphi \mid f: \leq u \in \mathbf{P}(S)\}
$$

denote its Orlicz- $\varphi$ family. (For more general functions $\psi$ we refer to $\mathbf{H}_{\psi}(S)$ as its Hardy- $\psi$ family.) To be sure $\mathbf{H}_{\varphi}(S)$ always contains the constant functions, but when it contains no other functions we say $\mathbf{H}_{\varphi}(S)$ is null. In the classic notation

$$
\oplus_{\varphi}=\left\{S: \mathbf{H}_{\varphi}(S) \text { is null }\right\}
$$

denotes the null Orlicz-p class of Riemann surfaces.

In particular if $\varphi_{p}(r) \equiv r^{p}$ then $\mathscr{C}_{\varphi_{p}}=\mathscr{C}_{p}(p>0)$ are the null Hardy- $p$ classes discussed by Heins. His basic result is that 


$$
\mathscr{\Theta}_{L A} \subset \bigcap_{q>0} \mathscr{\Theta}_{q} \subset \ldots \subset \bigcup_{q<p} \mathscr{\Theta}_{q} \subset \mathscr{\Theta}_{p} \subset \bigcap_{p<q} \mathscr{\Theta}_{p} \subset \ldots \subset \bigcup_{q>0} \mathscr{\Theta}_{q} \subset \mathscr{\Theta}_{B A},
$$

where $\mathscr{C}_{L A}, \mathscr{O}_{B A}$ is the null Lindelöfian analytic class, null bounded analytic class respectively. (Our set notation is that $A \subseteq B, A \subset B, A \notin B$ means inclusion, proper inclusion, no inclusion respectively and after Hausdorff $A \| B$ means $A \nsubseteq B$ and $B \neq A$.)

In the general case there are two growth conditions to be considered.

(4) Finite growth means $\lim \sup x^{-p} \varphi(x)<\infty$ for some $p>0$.

Infinite growth means $\lim \sup x^{-p} \varphi(x)=\infty$ for all $p>0$.

We shall call $\varphi$ superscalar when (5) holds and subscalar when (6) holds.

$$
\begin{gathered}
L_{\varphi}(r) \leq \varphi(K r), \\
\varphi(K r) \leq L \varphi(r),
\end{gathered}
$$

where in case (5) we, may choose

$$
K \rightarrow 0 \text { as } L \rightarrow 0 .
$$

In addition

$$
\begin{aligned}
\psi & \prec \varphi \text { means } \int_{0}^{\infty} \frac{\psi(\operatorname{inv} \varphi(t))}{1+t^{2}} d t<\infty, \\
\psi & \prec \prec \text { means } \int_{0}^{\infty} \frac{\psi[n(\operatorname{inv} \varphi(t))]}{1+t^{2}} d t<\infty, \text { for all } n>0,
\end{aligned}
$$

where inv $\varphi$ denotes the inverse of $\varphi$. Our main results are as follows:

Theorem 1. If $\varphi, \psi$ are Orlicz functions then

$$
\oplus_{\psi} \subset \mathscr{\Theta}_{\varphi} \text {, if } \psi \prec \prec \varphi \text {, }
$$

and if in addition $\varphi$ is superscalar or $\psi$ is subscalar then

$$
\mathscr{\Theta}_{\psi} \subset \mathscr{\Theta}_{\varphi} \text {, if } \psi \prec \varphi \text {. }
$$

Corollary 1. (Countable unions and intersections).

$$
\bigcup_{n=1}^{\infty} \mathscr{\Theta}_{\varphi_{n}} \subset \mathscr{\Theta}_{\varphi} \subset \bigcup_{m=1}^{\infty} \mathscr{\Theta}_{\varphi_{m}}
$$

whenever $\varphi_{n} \prec \prec \varphi \prec \prec \varphi_{m}$ or in the case that $\varphi, \varphi_{n}$ are superscalar or $\varphi_{n}, \varphi$ are subscalar whenever $\varphi_{m} \prec \varphi \prec \varphi_{m}$.

Corollary 2. (Arbitrary unions for finite superscalar functions).

$$
\bigcup_{\psi<\varphi} \mathscr{\Theta}_{\psi} \subset \mathscr{C}_{\psi},
$$

whenever $\varphi$ is finite and superscalar. 
Needless to say more questions are raised than answered. In particular the problem of characterizing the inclusion relations "ㄷ, $\subset$, $\notin$, II" between the $\mathscr{Q}_{\varphi}$ in terms of the relative growth relations between the $\varphi$ is left open.

The basic idea is to bring the theory of conformal strip mappings to bear on the problem of finding a replacement for $z^{p}$ in the general case. In particular the soft geometric extremal length distortion theorem of Jenkins-Oikawa [2] is used in the finite case and the hard analytic distortion theorem of Warschawski [3] is used in the infinite case. (It is interesting that both theorems were needed.) Once the replacements $h_{\varphi}$, which correspond to $\varphi$ and play the role of $z^{p}$ in the general case, are obtained Heins' methods may be applied.

I am grateful to David Drasin for his suggestions and especially for introducing me to the nuances in the theory of conformal strip mappings. I also wish to thank K. V. Rajeswara Rao and Gerald R. MacLane for their incisive remarks.

2. Conformal strip mappings. a. Although the results reviewed in this section hold for more general domains, it will suffice for our applications to restrict the study to symmetric strips with "nice" boundaries. A symmetric strip domain will be defined by

$$
S_{\theta}=\left\{x+i y:|y|<\frac{1}{2} \theta(x)\right\}
$$

where $\theta$ is positive and continuous. Let $w=f_{\theta}(z)$ denote the normalized (土 $\infty, 0$ are left fixed) conformal map of $S_{\theta}$ onto $S_{1}=\left\{u+i v:|v|<\frac{1}{2}\right\}$ and let $g_{\theta}(w)=z$ denote its inverse. The general problem is to describe the behavior of $f_{\theta}$ in terms of the behavior of $\theta$. Here we are interested in determining the conditions on $\theta$ under which $\left|\mathcal{R} f_{\theta}(x)-\Re f_{\Theta}(z)\right|$ is uniformly bounded, because this simulates the relation between $\left|z^{p}\right|$ and $r^{p}$. In the next section we shall show how to choose $\theta$ so that $R f_{\theta}(x)$ (weakly) approximates a prescribed $\log \varphi\left(e^{x}\right)$. In this section we point out two sufficient conditions on $\theta$ which guarantee that $2 f_{\theta}(z)=R f_{\theta}(x)+$ $+0(1)$. As we previously mentioned two types of distortion theorems are used.

A. The Jenkins-Oikawa Theorem. Although the theorem we have in mind is not explicitly stated as such in the Jenkins-Oikawa paper [2] it is a trivial consequence of their method and is certainly stated implicitly in their paper. We shall also outline their proof but first we need to recall some notation.

Let $Q_{\theta}(t)=\left\{0 \leq x \leq t,|y| \leq \frac{1}{2} \theta(x)\right\}$ and let any of the symbols $m_{\theta}(t)=m\left[Q_{\theta}(t)\right]=m \Gamma_{\theta}(t)$ denote the module for the family of arcs $\Gamma_{\theta}(t)$ in $Q_{\theta}(t)$ which separate the vertical sides of the quadrangle $Q_{\theta}(t)$. Let 


$$
\begin{gathered}
\underline{u}(x)=\inf _{y} \mathcal{R} f_{\theta}(z), \\
\bar{u}(x)=\sup _{y} \mathcal{R} f_{\theta}(z), \\
\operatorname{osc}(x)=\bar{u}(x)-\underline{u}(x) .
\end{gathered}
$$

The Jenkins-Oikawa approach to our problem depends on the initial observation that

$$
\left|R f_{\theta}(z)-m_{\Theta}(x)\right| \leq K
$$

if and only if $\operatorname{osc}(x) \leq B$. This follows immediately from the conformal invariance and monotonicity properties of modules, namely

$$
\begin{gathered}
m_{\theta}(x)=m\left[f_{\theta}\left(Q_{\theta}(x)\right)\right], \\
\underline{u}(x)-\bar{u}(0) \leq m\left[f_{\theta}\left(Q_{\theta}(x)\right)\right] \leq \bar{u}(x)-\underline{u}(0), \\
\bar{u}(x)-\underline{u}(0)=\underline{u}(x)-\bar{u}(0)+\operatorname{osc}(x)+\operatorname{osc}(0) .
\end{gathered}
$$

The module $m_{\theta}(x)$ can then be estimated by well-known techniques to give information about ' $\mathcal{R} f(z)$ such as Ahlfors' distortion theorem. But for our applications it will suffice to show that $m_{\theta}(x)$ itself has a desired behavior.

Consequently from our point of view their theorem is a sufficient condition on $\theta$ which guarantees $\operatorname{osc}(x) \leq B$. To state it let $L=L_{\theta}(x)>0$ be given, set $I=[x-L, x+L], \quad M=\sup \{\theta(t): t \in I\}, m=$ $\inf \{\theta(t): t \in I\}$ and define

$$
\mu_{\theta}(x)=\frac{L \min (M, L)}{[\min (m, L)]^{2}} .
$$

Then their theorem reads as follows:

Theorem A. (Jenkins-Oikawa). For a symmetric strip $S_{\theta}$ let $L_{\theta}(x)$ exist on a set $E \subset[0, \infty)$ and have the property that

$$
\mu_{\theta}(x) \leq B, \quad \text { for } x \in E .
$$

Then the mapping function $f_{\Theta}$ satisfies (15) at each $x \in E$ for $K$ independent of $x$.

The proof is delayed until we state the next theorem.

B. The Warschawski Theorem. The actual result we quote is Warschawski's Theorem [3] but it is clear how this originated from Ahlfors' distortion theorem (see [3]). Warschawski calls the stip $S_{\Theta}$ an L-strip (of inclination zero) if $\left(\theta\left(x_{2}\right)-\theta\left(x_{1}\right)\right) /\left(x_{2}-x_{1}\right) \rightarrow 0$ as $x_{2}, x_{1} \rightarrow \infty$. As we shall see the degree of smoothness of $\theta$ makes no difference for our applicatons so we shall assume $\theta^{\prime}(t)$ is continuous. L-trip condition is $\theta^{\prime}(t) \rightarrow 0$ as $t \rightarrow \infty$. 
Theorem B. (Warschawski). If $S_{\theta}$ is a smooth symmetric L-strip and

$$
\int_{0}^{\infty} \frac{\left(\theta^{\prime}(t)\right)^{2}}{\theta(t)} d t<\infty
$$

then $f_{\theta}$ has the asymptotic expansion

$$
f_{\theta}(z)=\lambda+\int_{0}^{x} \frac{d t}{\theta(t)}+i \frac{y}{\theta(x)}+0(1)
$$

where $\lambda$ is a real constant.

(Actually, Warschawski's original formula is slightly more complicated because it also includes the non-symmetric case.)

b. We outline the proofs. Because $m_{\Theta}(x)=m\left[f_{\theta}\left(Q_{\theta}(x)\right)\right]$ we let

$R_{\theta}(x)=\left\{\begin{array}{l}\left\{\bar{u}(0)-1 \leq u \leq u(x)+1,|v| \leq \frac{1}{2}\right\}, \text { if } \underline{u}(x)-\bar{u}(0)>-1, \\ \left\{\bar{u}(0)-1 \leq u \leq \bar{u}(0), \quad|v| \leq \frac{1}{2}\right\}, \quad \text { if } \quad \underline{u}(x)-\bar{u}(0) \leq-1,\end{array}\right.$

and set $\varrho(w)=1$ in $R_{\theta}(x)$, zero elsewhere. Then $\varrho(w)|d w|$ is admissible for the module $m\left[f_{\theta}\left(Q_{\theta}(x)\right)\right]$ and hence

$$
m_{\theta}(x) \leq\left\{\begin{array}{l}
\underline{u}(x)-\bar{u}(0)+2, \text { if } \underline{u}(x)-\bar{u}(0)>-1, \\
1, \text { if } \underline{u}(x)-\bar{u}(0) \leq-1 .
\end{array}\right.
$$

Next let $l_{t}=\left\{x=t,|y| \leq \frac{1}{2} \theta(t)\right\}$ denote the vertical line segment cut off by $S_{\theta}$ and note that $B_{\theta}(x)=\left\{l_{t}: 0 \leq t \leq x\right\} \subset \Gamma_{\theta}(x)$. Therefore by monotonicity $m_{\theta}(x)=m \Gamma_{\theta}(x) \geq m B_{\theta}(x)$ and since $[\theta(x)]^{-1}|d z|$ is extremal for the last module $m B_{\theta}(x)$, we obtain

$$
\begin{gathered}
m B_{\theta}(x)=\iint_{Q_{\theta}(x)} \theta^{-2} d x d y=\int_{0}^{x} \theta^{-1} d x \text { and hence } \\
m_{\theta}(x) \geq \int_{0}^{x} \frac{d t}{\theta(t)} .
\end{gathered}
$$

Hence as soon as Ahlfors' condition $\int_{0}^{x}[\theta(t)]^{-1} d t>1$ holds we must have $m_{\ominus}(x)>1$ and hence $\underline{u}(x)-\bar{u}(0)>1$ must hold. This proves the Ahlfors distortion theorem.

Now to get the upper inequality we note that $T_{\Theta}(x)=\left\{\underline{u}(0) \leq u \leq \bar{u}(x), \quad|v| \leq \frac{1}{2}\right\}$ has module $m T_{\theta}(x)=\bar{u}(x)-\underline{u}(0)$ and hence by conformal invariance 


$$
\bar{u}(x)-\underline{u}(0)=m\left[g_{\theta}\left(T_{\theta}(x)\right)\right] .
$$

Then we use a variation of the previous trick to estimate $m\left[g_{\theta}\left(T_{\theta}(x)\right)\right]$. Let $\varrho_{*}(z)|d z|$ denote the extremal metric for $m_{\theta}(x)$. Then if $L_{\theta}(x)$ is given set $\varrho_{s}=\left[\min \left(L_{s}, m_{s}\right)\right]^{-1}$ on $\left\{|x-s| \leq L_{s},|y| \leq \frac{1}{2} \min \left(L_{s}, M_{s}\right)\right\}$, zero elsewhere, for $s=0, x$. Thus if

$$
\varrho(z)=\max \left(\varrho_{0}(z), \varrho_{*}(z), \varrho_{x}(z)\right)
$$

then $\varrho(z)|d z|$ is admissible for $m\left[g_{\theta}\left(T_{\theta}(x)\right)\right]$ and we obtain

$$
m\left[g_{\theta}\left(T_{\theta}(x)\right)\right] \leq \mu_{\theta}(0)+m_{\theta}(x)+\mu_{\theta}(x),
$$

which because of (21), (20), (17) completes the proof of Theorem A.

To get Warschawski's upper inequality recall that if $\omega \in C^{1}\left(Q_{\theta}(x)\right)$ and if $\omega\left(x, \frac{1}{2} \theta(x)\right) \equiv 1, \quad \omega\left(x,-\frac{1}{2} \theta(x)\right)=0, \quad|\operatorname{grad} \omega||d z| \quad$ is admissible for $m_{\theta}(x)$ and hence with $\omega(x, y)=\frac{1}{2}+(y / \theta(x))$ on $Q_{\theta}(x)$, zero elsewhere observe that

$$
m_{\theta}(x) \leq \iint_{Q_{\theta}(x)}|\operatorname{grad} \omega|^{2} d x d y=\int_{0}^{x} \frac{d t}{\theta(t)}+\frac{1}{12} \int_{0}^{x} \frac{\left(\theta^{\prime}(t)\right)^{2}}{\theta(t)} d t,
$$

which is part of Warschawski's inequality. (In the non-symmetric case where $\quad S=\left\{\emptyset_{-}(x)<y<\varnothing_{+}(x)\right\}, \quad \theta(x)=\emptyset_{+}(x)-\varnothing_{-}(x), \quad \psi(x)=$ $\frac{1}{2}\left(\varnothing_{+}(x)+\emptyset_{-}(x)\right)$, and $\varnothing_{ \pm}$are continuously differentiable use $\omega(x, y)=$ $\frac{1}{2}+(y-\psi(x)) / \theta(x)$ to obtain

$$
m_{\theta}(x) \leq \int_{0}^{x} \frac{1+\left(\psi^{\prime}(t)\right)^{2}}{\theta(t)} d t+\frac{1}{12} \int_{0}^{x} \frac{\left(\theta^{\prime}(t)\right)^{2}}{\theta(t)} d t .
$$

The expansion holds in the non-symmetric case when $\lim \emptyset_{+}^{\prime}=\lim \emptyset_{-}^{\prime}=0$,

$$
\begin{gathered}
\int_{0}^{x}\left(\theta^{\prime}(t)\right)^{2}(\theta(t))^{-1} d t<\infty \quad \text { with } \int_{0}^{x}(\theta(t))^{-1} d t \text { replaced by } \\
\left.\int_{0}^{x}\left[1+\left(\psi^{\prime}(t)\right)^{2}\right](\theta(t))^{-1} d t .\right)
\end{gathered}
$$

Finally to obtain the asymptotic expansion of Warschawski we appeal to Warschawski [3].

c. In the case of finite growth we mentioned that $m_{\theta}(x)$ will be chosen to approximate $\operatorname{lcg} q\left(e^{x}\right)$ for $\theta$ appropriately chosen. This will be acecroplished by a continuity argurent, for which we prepare by establishing a simple fact about $m_{\theta}(x)$. For that purpose let 


$$
\left\|f_{1}-f_{2}\right\|_{x}=\sup \left\{\left|f_{1}(t)-f_{2}(t)\right|: 0 \leq t \leq x\right\}
$$

be the uniform metric.

Continuity Lemma 1. Suppose $\psi, \theta$ are positive continuous with $\psi$ fixed. Then $\left\|m_{\theta}-m_{\psi}\right\|_{x} \rightarrow 0$ as $\|\theta-\psi\|_{x} \rightarrow 0$.

The modules $m_{\theta}(t)$ are determined by (or according to Grötzsch defined by) the unique conformal maps $w=F_{\theta, t}(z)$ of $Q_{\theta}(t)$ onto $R_{\theta}(t)=$ $\left\{0 \leq v \leq 1, \quad 0 \leq u \leq m_{\theta}(t)\right\}$. As $\|\theta-\psi\|_{t} \rightarrow 0$ the domains converge $Q_{\theta}(t) \rightarrow Q_{\psi}(t)$ in the sense of Carathéodory. By Montel's theorem a subsequence of the $F_{\theta, t}$ converge, by Carathéodory theorem, to a conformal map $F_{0}$ of $Q_{\psi}(t)$ onto a rectangle $R_{0}=\{0 \leq v \leq 1,0 \leq u \leq m\}$. By uniqueness of the defining conformal map $m=m_{\theta}(t), F_{0}=F_{\theta}, t$ and the lemma follows.

3. Weak approximation. a. Let us assume that $\varphi, \psi$ are Orlicz functions. It is a trivial but useful result that

$$
\mathscr{\Theta}_{\varphi}=\mathscr{\Theta}_{\psi} \text {, if } K^{-1} \varphi \leq \psi \leq K \varphi \text {, }
$$

for some $K>0$, where $f \leq g$ means $f(x) \leq g(x)$ for $x \leq x_{0}$. Now since $\Phi(x)=\varphi\left(e^{x}\right)$ is convex and increasing, we may write $\Phi(x)=\int_{0}^{x} \Theta(t) d t$, where $\Theta(t)$ is non-decreasing and, since $x^{-1} \Phi(x) \rightarrow \infty$ as $x \rightarrow \infty$ we must have $\Theta(x) \rightarrow \infty$ as $x \rightarrow \infty$. Thus we may approximate $\Theta(x)$ by a positive $C^{\infty}$ function $\Theta_{1}(x)$ such that

$$
\begin{aligned}
& \frac{1}{2} \Theta(x) \leq \Theta_{1}(x) \leq 2 \Theta(x), \\
& \Theta_{1}^{\prime}(x)>0 .
\end{aligned}
$$

Hence if $\Phi(x)=\int_{0}^{x} \Theta_{1}(t) d t$ then $\frac{1}{2} \Phi(x) \leq \Phi_{1}(x) \leq 2 \quad \Phi(x)$ and $\Phi_{1}^{\prime \prime}(x)$ is positive and continuous. Finally by Arakelyan's fundamental theorem of approximation or perhaps by more elementary theorems there is a real analytic function $F(x)$ such that $\frac{1}{2} \Phi_{1}^{\prime \prime}(x) \leq F(x) \leq 2 \Phi_{1}^{\prime \prime}(x)$. Consequently $\Phi_{2}(x)=\int_{0}^{x}\left(\int_{0}^{t} F(u) d u\right) d t$ satisfies $\frac{1}{2} \Phi_{1} \leq \Phi_{2} \leq 2 \Phi_{1}$. Hence by (23) we obtain the following fact

Lemma 2. The degree of smoothness of $\varphi$ does not affect the classification $\mathscr{Q}_{\varphi}$. In other words for each Orlicz function $\varphi$ there is a real analytic Orlicz function $\psi$ such that $\mathscr{\Theta}_{\psi}=\bigotimes_{\psi}$.

b. The main tool for constructing the functions $h_{\varphi}$ which correspond to $\varphi$ and play the role of $z^{p}$ is the following

Weak approximation lemma 3. Let $\lambda(x)$ denote an orientation preserving homeomorphism of $(-\infty, \infty)$ onto itself for which $\log x=o(\lambda(x))$ 
for $x$ large. Then there exists a positive continuous function $\theta=\theta_{\varphi}(x)$ such that the symmetric strip $S_{\theta}$, the normalized conformal strip mapping $f_{\theta}$, of $S_{\theta}$ onto $S_{1}$, and its inverse $g_{\theta}$ have the following properties:

$$
\begin{aligned}
& \mathscr{R} f_{\theta}(z) \geq \lambda(x)-K, \\
& g_{\theta}\{|v| \leq a\} \supseteq \bigcup_{n=1}^{\infty}\left\{|y| \leq b, x=b_{n}\right\}, \\
& \Re f(z) \leq \lambda(x)+K, \text { for } x=b_{n},
\end{aligned}
$$

where $K>0,0<a, b<\frac{1}{2}, b_{n}{ }^{\nearrow} \infty$.

The proof will be in two cases according to whether or not $\lambda(x) \leq p x$ for some $p>0, x \leq x_{0}$, which we refer to as finite or infinite growth respectively.

Case A. (finite growth). Since $\lambda(x) \leq p x$ we may choose $\theta(t)=p^{-1}$. Then $m_{\theta}(x)=p(x) \geq \lambda(x)$, for $x \leq x_{0}$. On the other hand if we choose $\theta(t) \equiv t$ then $m_{\theta}(x) \equiv \log x<\lambda(x)$ for $x \geq x_{0}$. We shall push up on the graph of $\theta \equiv p^{-1}$ from below causing a »dent» to form and thereby causing $m_{\ominus}(x)$ to decrease. Condition (24) will be maintained throughout the deformation which is used to obtain condition (26). For functions $\theta(x)$ with $\theta(x) \equiv \theta(a)$, for $a \leq x \leq b$, we define the dent deformation $D_{\mathrm{a}, \mathrm{b}}(\theta)=\chi$, as follows:

$$
\chi(x)= \begin{cases}\theta(x), & \text { if } x \leq a \text { or } x \leq b, \\ \theta(a)+(x-a), & \text { if } a \leq x \leq c, \\ \theta(a)+(c-a)-(x-c), & \text { if } c \leq x \leq b,\end{cases}
$$

where $c=\frac{1}{2}(a+b)$. Now clearly $m_{\chi}(x) \searrow$ as $b \nearrow$ continuously by the continuity lemma 1 . Hence there is a first $b$ for which both

$$
\begin{gathered}
m_{x}\left(x_{0}\right)=\lambda\left(x_{0}\right), \\
m_{x}(x) \geq \lambda(x),
\end{gathered}
$$

for $x \geq x_{0}$. Then take $a, b$ much larger and repeat the dent deformation to obtain the desired $\theta$ by induction.

To see that $g_{\theta}$ has the weak covering property (25) we note first that by Carathéodory's theorem $g_{\theta}$ may be assumed to be homeomorphic on the closed strip $S_{1}$. Then by the fact that $\theta$ was formed by the dent deformation from $\theta(x) \equiv p^{-1}$ we have

$$
9 g_{\theta}(u+v i) \geq 9\left(p^{-1}(u+v i)\right)
$$

on $\partial S_{1}=\left\{v \equiv \pm \frac{1}{2}\right\}$ and hence throughout $S_{1}$. Hence condition (25) is obtained and in the finite growth case we obtain the strong covering property 


$$
g_{\Theta}\{|v| \leq a\} \supset\left\{|y| \leq a p^{-1}\right\} .
$$

To see that (24), (26) hold we need only apply the Jenkins-Oikawa theorem $A$ to show that $\mathscr{R} f_{\theta}(z)=m_{\theta}(x)+0(1)$. It suffices to check condition (17). With $E=[0, \infty), L=\theta(x)+1 \geq p^{-1}+1,(p<1)$, we find that $\mu_{\theta}(x) \leq\left(\frac{1+p}{1-p}\right)^{2}$ in (17).

Case B. (Infinite growth). We assume $\theta\left(x_{p}\right)>p x_{p}$, for all $p>0$, $x_{p} \geq K$, for $K$ given. In other words if $\chi \equiv p^{-1}$ then $\theta\left(x_{p}\right)>m_{\chi}\left(x_{p}\right)$. To prove this case we shall apply the Warschawski Theorem B to the resulting $\theta$ in the conclusion of the following

Lemma 4. Given $\lambda$ as in the hypothesis of lemma 3, there is a positive continuous differentiable function $\theta$ with the following properties:

$$
\int_{0}^{x} \frac{d t}{\theta(t)} \geq \lambda(x)-K,
$$

$$
\theta\left(b_{n}\right) \geq b
$$

$$
\int_{0}^{b_{n}} \frac{d t}{\theta(t)} \leq \lambda\left(b_{n}\right)+K,
$$

$$
\lim \theta^{\prime}(t)=0
$$

$$
\int_{0}^{\infty} \frac{\left(\theta^{\prime}(t)\right)^{2}}{\theta(t)} d t<\infty
$$

where $K>0,0<b, b_{n} \nearrow \infty$.

Proof of Lemma 4: Consider $\tau(x)=\exp (-1 / \mathrm{x})$, for $0 \leq x \leq 1$, where $\tau(0)=0$ is understood and set $\tau_{n}(x)=\tau\left(4^{-n} x\right)$, for $0 \leq x \leq 4^{n}$. Notice that:

$$
\begin{aligned}
\left|\tau_{n}^{\prime}(x)\right| & \leq 4^{-n}\left(4 / e^{2}\right) \\
\tau_{n}\left(4^{n}\right) & =e^{-1} \\
\int_{0}^{4^{n}} \frac{\left(\tau_{n}^{\prime}(t)\right)^{2}}{\tau_{n}(t)} d t & =4^{-n} \int_{0}^{1} t^{-4} e^{-1 / t} d t .
\end{aligned}
$$

Then choose $\delta_{n}$ so small that 


$$
\frac{2 \cdot 4^{n}}{\tau\left(\delta_{n}\right)} \geq \lambda\left(4^{n+2}\right)
$$

Now define $\theta$ initially by the formula

$$
\theta(x)= \begin{cases}\tau\left(\delta_{n}\right), & \text { if } 4^{n} \leq x \leq 2 \cdot 4^{n}+4^{n} \delta_{n}, \\ \tau\left(\left[x-\left(2 \cdot 4^{n}\right)\right] 4^{-n}\right), & \text { if } 2 \cdot 4^{n}+4^{n} \delta_{n} \leq x \leq 3 \cdot 4^{n}, \\ \tau\left(\left[4^{n+1}-x\right] 4^{-n}\right), & \text { if } 3 \cdot 4^{n} \leq x \leq 4^{n+1}-4^{n} \delta_{n+1}, \\ \tau\left(\delta_{n+1}\right), & \text { if } 4^{n+1}-4^{n} \delta_{n+1} \leq x \leq 4^{n+1} .\end{cases}
$$

Evidently properties (28), (29), (31), (32) hold. To get property (30) we define the push deformation $P_{\mathrm{a}, \mathrm{b}}(\theta)=\chi$ as follows:

$$
\chi(x)= \begin{cases}\theta(x), & \text { if } x \leq a, \\ \theta(a), & \text { if } a \leq x \leq b, \\ \theta(x-(b-a)), & \text { if } b \leq x .\end{cases}
$$

If (30) holds then there is nothing to do. If (30) fails to hold then it still fails after a push deformation. The other 4 properties continue to hold. However because $\lambda$ has infinite growth characteristics there is a first $b=b_{1}$ for which $\psi=P_{\mathrm{a}, \mathrm{b}}(\theta)$ satisfies (30) at $b_{1}$. Consequently subsequent push deformations for $a, b$ much larger will ensure that $\theta$ can be constructed with the 5 properties of lemma 4 .

Proof of Lemma 3: Consider $\theta$ with the properties of lemma 4. By (31) $S_{\theta}$ is an $L$-strip and by (32) Warschawski's expansion (19) holds. Hence properties (24), (25), (26) can be read from properties (28), (29), (30) by Warschawski's expansion.

c. The reason for the dichotomy in this proof is that there is a dichotomy in the theory of conformal strip mappings. In the finite case, conditions (31), (32) may not be possible, which makes it difficult to apply Warschawski's theorem. In the infinite case it is difficult to obtain the weak covering property without the help of Warschawski's $L$-strip theory.

d. We conclude this section by describing the functions $h_{\varphi}$ which play the role of $z^{p}$. The only problem will be to define $\lambda$. There are several cases.

I. The superscalar case. Given a superscalar Orlicz function $\varphi$, set

$$
\lambda(x)=\log \varphi\left(e^{x}\right) \text {. }
$$

II. The subscalar case. Let $\psi$ be a subscalar Orlicz function, $\varphi$ an Orlicz function and $\psi \prec \varphi$. Then for every $n>0$

$$
\int_{0}^{\infty} \frac{\psi[n(\operatorname{inv} \varphi(t))]}{1+t^{2}} d t \leq M_{n} \int_{0}^{\infty} \frac{\psi(\operatorname{inv} \varphi(t))}{1+t^{2}} d t<\infty .
$$


Hence $a_{n} \nearrow \infty$ may be found such that

$$
\int_{a_{n}}^{\infty} \frac{\psi(n \operatorname{inv} \varphi(t))}{1+t^{2}} d t<\frac{1}{2^{n}} .
$$

For $b_{n}=n \operatorname{inv} \varphi\left(a_{n}\right)$ select the $a_{n}$ to diverge so rapidly that an Orlicz. function $\chi$ can be found with the properties:

$$
\begin{array}{ll}
\chi(r) \geq \varphi(r / n), & \text { if } b_{n} \leq r \leq b_{n+1}, \\
\chi(r) \leq \varphi(r / n), & \text { if } r \leq b_{n+2},
\end{array}
$$

then set

$$
\lambda(x)=\log \chi\left(e^{x}\right) .
$$

In this case $\lambda$ depends on $\psi$ and $\varphi$.

III. General case. If $\psi \prec \prec \varphi$ then (37) holds and we proceed to choose $\chi$ and $\lambda$ exactly as in case II.

The crucial property. In case I let $\psi \prec \varphi$, in case II, III $\psi$ is given. Let $\chi=\varphi$ in case I. Then in every case

$$
\int_{0}^{\infty} \frac{\psi(\operatorname{inv} \chi(t))}{1+t^{2}} d t<\infty .
$$

Approximation. Given $\lambda=\lambda_{\varphi}$ defined in case I, II, III we obtain $\theta=\theta_{\varphi}$ from the weak approximation lemma 4. (In case II, III $\theta$ also depends on $\psi$.) Then the symmetric strip $S_{\theta}$ and the conformal functions $f_{\Theta}, g_{\theta}$ are defined with properties $(24),(25),(26)$. Let $\zeta$ be the variable in $S_{\theta}$ and let $\omega$ be the variable in $S_{1}$. Define

$$
A_{\varphi}=\left\{(z, \zeta): \zeta \in S_{\theta}, \quad z=e^{\zeta}\right\} .
$$

Then the defining parameter $\tau:(z, \zeta) \rightarrow \zeta$ gives $A_{\varphi}$ a conformal structure and the canonical projection $\pi:(z, \zeta) \rightarrow z$ defines $A_{\varphi}$ as a smooth covering surface over the complex plane. We then define the conformal equivalence between $A_{\varphi}$ and the halfplane $H=\left\{R_{\omega}>0\right\}$ by

$$
\begin{aligned}
h_{\varphi}(z, \zeta) & =\exp f_{\theta}(\zeta), \\
k_{\varphi}(\omega) & =\left(\exp g_{\theta}(\log \omega), g_{\theta}(\log \omega)\right) .
\end{aligned}
$$

From (24), (25), (26) we obtain

$$
\begin{aligned}
\left|h_{\varphi}(z, \zeta)\right|=\chi(|z|) / K, \\
\pi\left[k_{\varphi}\{\arg \omega \mid \leq b\}\right] \supseteq \bigcup_{n=1}^{\infty}\left\{|\arg z| \leq b_{n},|z|=b_{n}\right\},
\end{aligned}
$$




$$
\left|h_{\varphi}(z, \zeta)\right| \leq \chi(|z|) K, \text { if }|z|=b_{n},
$$

where $K>0,0<a, b<\frac{\pi}{2}, b_{n} \nearrow \infty$. If in addition $\varphi$ is finite and subscalar then the strong covering property holds

$$
\pi\left[k_{\varphi}\{|\arg \omega| \leq a\}\right] \supseteq\{|\arg z| \leq b\} .
$$

This follows from the strong covering property (27) for $g_{\theta}$.

The crucial property again. In every case I, II, III we have

$$
\int_{-\infty}^{\infty} \frac{\psi\left|k_{\varphi}(i t)\right|}{1+t^{2}} d t<\infty .
$$

That is because $\left|h_{\varphi}(z, \zeta)\right| \geq \chi(|z|) / K$ implies $\left|k_{\varphi}(w)\right| \leq \operatorname{inv} \chi(K|w|)$ and hence (48) follows from (41).

4. Construction of surfaces. a. The surfaces $S$ we construct are essentially a hybrid of two of Heins' examples. We "tear» the "Riemannian sectors» from an example [1, III, $5, \mathrm{p} .47]$, deform it into the isector» $A_{\varphi}$ described in section $3 \mathrm{~d}$ and "weld" these back onto another of his examples [1, III, $3, p .37]$. The "sectors» ensure that the surface $S \in \mathscr{C}_{p}$ and the "base surface» ensures that $S \notin \mathscr{C}_{w}$.

b. Given the Orlicz function $\varphi$ we choose $A_{p}, h_{\varphi}, k_{\varphi}$ as in section $3 \mathrm{~d}$ according to case I, II, or III. Only in the first case ( $\varphi$ subscalar), are these actually independent of $\psi$. In the other two cases they depend on $\psi \prec \varphi, \psi \prec \prec \varphi$. Considering the covering number $b>0$ in property (44), choose an integer $N>2 \pi / b$ and denote the $N t h$ roots of unity by $\omega_{v}=\exp (2 \pi i v / N)$ for $1 \leq v \leq N$. Then define the marked disks and marked sectors as follows:

$$
\begin{array}{ll}
D_{1}=\{(z, 1):|z|<3\}, & \text { for } n=1, \\
D_{2}=\{(z, 2):|z|<3\}, & \text { for } n=2, \\
D_{n}=\{(z, n):|z|<n\}, & \text { for } n \geq 3, \\
B_{v}=\left\{(z, w, v): z=\omega_{v} \pi g_{\theta}(w)\right\}, & \text { for } 1 \leq v \leq N .
\end{array}
$$

We abuse the notation by defining the canonical projection $\pi$ as the projection of an $n$-tuple onto its first coordinate (for example $\pi(z, w, v)=z$, $\pi(z, n)=z)$. We shall attempt to indicate what is the topological structure of the surface described in each case. Then the conformal structure will always be induced by the canonical projection $\pi$. For example $D_{n}, A_{v}$ are bordered covering surfaces because $\pi$ is univalent on them.

We shall first "puncture» and "cut» these marked surfaces and then at pairs of "cuts» which have common projections "weld» them together by the usual "criss-cross" technique. To define the cuts let $a_{v}=2^{-2 v}, b_{v}=$ 
$2^{-2 v+1}, \quad c_{v}=3-2^{-2 v}$ be fixed so that $b_{v}>a_{v}>b_{v+1} \ngtr 0$, and choose $d_{v}$ arbitrary (for the moment) except that

$$
2<\ldots<c_{v}<d_{v}<c_{v+1} \nearrow 3 .
$$

Designate the cuts as follows:

$$
\begin{aligned}
I_{v} & =\left\{\left(\omega_{v} t, w, v\right) \in A_{v}: 1 \leq t \leq 2\right\}, & & (1 \leq v \leq N), \\
I_{v 2} & =\left\{\left(\omega_{v} t, 2\right) \in D_{2}: 1 \leq t \leq 2\right\}, & & (1 \leq v \leq N), \\
J_{v n} & =\left\{(t, n) \in D_{n}: a_{v} \leq t \leq b_{v}\right\}, & & (v \geq 1, n=1,2), \\
K_{v n} & =\left\{(t, n) \in D_{n}: c_{v} \leq t \leq d_{v}\right\}, & & (v \geq 3, n=2 \text { or } v=n \geq 3) .
\end{aligned}
$$

The punctured and cut disks and sectors are then defined as follows:

$$
\begin{array}{ll}
E_{1}=D_{1}-\{(0,1)\}-\bigcup_{v=1}^{\infty} J_{v 1} \\
E_{2}=D_{2}-\{(0,2)\}-\bigcup_{v=1}^{\infty} J_{v 2}-\bigcup_{v=1}^{N} I_{v 2}-\bigcup_{v=3}^{\infty} K_{v 2}, \\
E_{n}=D_{n}-K_{n n}, & (n \geq 3), \\
A_{v}=B_{v}-I_{v} & (1 \leq v \leq N) .
\end{array}
$$

To repeat $E_{n}, A_{v}$ are considered as bordered Riemann covering surfaces of the complex plane $C$ with projection $\pi$.

c. To form a Riemann surface from these pieces it is only necessary to explain how to identify border points on cuts with common projections under $\pi$. The topology will then be given by the quotient topology and the conformal structure will be determined by $\pi$. To explain the identification we orient the cuts $I$ so that in their positive direction the modulus of their $\pi$-projection increases. Hence it makes sense to speak of the right or left side of a cut $I$ and to speak of the right, left border point $p_{+}, p_{-}$respectively which corresponds to the interior point $p$ of the cut $I$.

The repair weld at a cut $I$ is the surface formed by identifying border points the of the cut $I$ which have a common projection

$$
p_{+}=p_{-} .
$$

The criss-cross weld between two cuts $I_{1}, I_{2}$ which have a common projection is formed by first identifying end points $p_{1}=p_{2}$ when $\pi\left(p_{1}\right)=$ $\pi\left(p_{2}\right)$, then at interior points

$$
\left\{\begin{array}{l}
p_{1^{+}}=p_{2^{-}} \\
p_{1^{-}}=p_{2^{+}}
\end{array}\right.
$$

when $\pi\left(p_{1}\right)=\pi\left(p_{2}\right)=\pi\left(p_{1 \pm}\right)=\pi\left(p_{2 \pm}\right)$. 
Now the primary surface $S_{\varphi}$ is formed by criss-cross welding all pieces $E_{v}(v \geq 1)$ and $A_{v}(1 \leq v \leq n)$ at all pairs of cuts with common projections. (There are never more than two cuts with a common projection.) The $n$th truncation is

$$
S_{\varphi}^{n}=S_{\varphi}-\bigcup_{\nu=n+1}^{\infty} E_{\nu} \quad(n \geq 2)
$$

and the repaired $n$th truncation $T_{\varphi}^{n}$ is obtained from $S_{\varphi}^{n}$ by using the repair weld at $I_{v 2}$ for $v \geq n+1$.

d. In this subsection the basic properties of $S_{\varphi}, S_{\varphi}^{n}, T_{\varphi}^{n}$ are discussed. Evidently they are all ramified covering surfaces over $C$ with projection $\pi$. The covering is smooth except at the end points of the cuts $I$, where there is a criss-cross weld, these last points being branch points of order $2-1=1$. We may designate them by $S, S_{\varphi}, S_{\varphi, \psi}$, etc., according to the degree of dependence we wish to denote. For similar reasons we may wish to write $\pi=\pi_{p}=\pi_{\varphi, \psi}$.

The basic coverage property is

$$
\begin{aligned}
\pi\left(S_{\varphi}\right) & =C, & & \text { in general, } \\
\pi\left(T_{\varphi}^{3}\right) & =C, & & \text { when } \varphi \text { has finite growth. }
\end{aligned}
$$

It is also useful to note that

$$
S_{\varphi}^{n} \subset S_{\varphi}^{n+1} \subset \ldots \subset \bigcup_{n=3}^{\infty} S_{\varphi}^{n}=S_{\varphi} .
$$

We adapt Heins' notation $M f$ as the least harmonic majorant of the (real valued) function $f$ on its domain of definition $D$. Since $M f$ depends on $D$ we may wish to write $M f / D=M f$ in order to avoid confusion. With this notation we state the basic Myrberg property that if $f \in \mathbf{A}(S)$, where $S=S_{\varphi}$, or $T_{\varphi}^{3}$ has property (52) or (53), $\chi$ is any Orlicz function, and $M \chi|f| / S$ exists, then

$$
f=g \circ \pi, \quad \text { where } g \in \mathbf{A}(C) .
$$

To prove this we resort to Heins' variation of the Myrberg argument [1, Remark, p. 37]. Restrict $f(p)$ to

$$
\left.R=\left(S_{\varphi}-\bigcup_{v=3}^{\infty} E_{v}-\bigcup_{v=1}^{N} A_{v}\right) \bigcap\left\{p:|\pi(p)|<\frac{1}{2}\right\}\right]
$$

and consider

$$
F(z)=\left[\frac{f(p)-f(q)}{2}\right]^{2}, \text { where }\{p, q\}=\pi^{-1}(z) .
$$


Note that $F$ is analytic on $\left\{0<|z|<\frac{1}{2}\right\}$ and

$$
F\left(z^{-n}\right)=0 \text {. }
$$

Now $\chi\left(e^{x}\right)=\Phi(x)$ has the Orlicz and de La Vallée Poussin conditions (1), (2) and $\frac{1}{2} \log |F|$ is subharmonic. Moreover $\chi|f| \leq u \in \mathrm{P}(S)$. It follows that $\left.\left.\chi\right|^{F}\right|^{1 / 2} \leq U$ harmonic on $\left\{0<|z|<\frac{1}{2}\right\}$. Hence by the SzegöSolomentsev Theorem [1, p. 17] $\left.M_{\chi}\right|^{|F|^{1 / 2}}$ is quasi-bounded and hence constant on $\left\{0<|z|<\frac{1}{2}\right\}$. Since $F$ is bounded its singularity at $z=0$ is removable and since $F\left(2^{-n}\right)=0, F(z) \equiv 0$ by the accumulationof-zeros principle.

Hence $f(p) \equiv f(q)$ on $R$ whenever $\pi(p)=\pi(q)$. Proceeding to argue along similar lines by analytic continuation and the accumulation of zeros principle at the cuts $I$ we obtain that $f(p)=f(q)$ for $p, q \in S$ whenever $\pi(p)=\pi(q)$. By the basic coverage properties (52) or (53) property (54) follows.

Each sector $B_{v}$ has an associated conformal map $h_{v}$ defined by

$$
h_{\nu}(z, \zeta, v)=h_{\varphi}\left(\bar{\omega}_{v} z, \zeta\right)
$$

and a conformal inverse $k_{v}$ on $H$. These maps have properties similar to $(44),(45),(46)$.

5. Proof of the main result. a. We have constructed the surfaces $S_{\varphi}$. We first prove

Lemma 5. $S_{\varphi} \in \bigoplus_{\varphi}$.

Suppose $f \in \mathbf{H}_{\varphi}\left(S_{\varphi}\right)$. Then $f \in \mathbf{A}\left(S_{\varphi}\right)$ and $\varphi|f| \leq u \in \mathbf{P}\left(S_{\varphi}\right)$. Restrict this to $A_{v}$ and change variables by $k_{v}$ to obtain on $H-h_{\nu}\left(I_{v}\right)$

$$
\varphi\left|f\left(k_{\nu}(w)\right)\right| \leq u\left(k_{\nu}(w)\right)=U(w) .
$$

Now $f \circ k_{v} \in \mathbf{A}\left(H-h_{\nu}\left(I_{v}\right)\right.$ so $u=\log \left|f \circ k_{\nu}\right|$ is subharmonic. Moreover $\Phi(x)=\varphi\left(e^{x}\right)$ has properties (1), (2) and $U \in \mathbf{P}\left(H-h_{\nu}\left(I_{v}\right)\right)$ therefore the Szegö-Solomentsev theorem implies that $M \Phi u=M \varphi\left|f \circ k_{v}\right|$ is quasi-bounded. Consequently, $M_{\varphi}\left|f \circ k_{v}\right|$ has a Poisson representation on $\{c i w>c\}$ and hence

$$
\varphi\left|f\left(k_{v}(w)\right)\right| \leq \varepsilon|w|
$$

for $|w| \geq M_{\nu}(\varepsilon), \quad|\arg w| \leq b$. Change variables back by $w=h_{\nu}(p)$, use the representation (54) that $f=g \circ \pi$ and the weak covering property (44) to obtain

$$
\varphi|g(z)| \leq \varepsilon \max _{\nu}\left|h_{\varphi}\left(\bar{w}_{\nu} z, \zeta\right)\right|,
$$

for $|z|=b_{n} \geq \mathcal{R}$. Now if $\varphi$ is subscalar $\varepsilon\left|h_{\varphi}(z)\right| \leq \varepsilon K \varphi(|z|) \leq \varphi(\delta(\varepsilon)|z|)$. Otherwise $\varepsilon\left|h_{\varphi}(z)\right| \leq \varepsilon \varphi\left(\frac{1}{n}|z|\right) \leq \varphi(\delta(\varepsilon)|z|)$ by choice of $h_{\varphi}$. Therefore in any case 


$$
\varphi|g(z)| \leq \varphi(\delta(\varepsilon)|z|),
$$

for $|z|=b_{n} \geq R$. Consequently, by taking inv $\varphi$ of both sides, applying the maximum modulus principle and Liouville's Theorem we find that $g(z)$ is constant. Since $f=g \circ \pi$, it is also constant.

b. It remains only to choose the sequence $\left\{d_{v}\right\}$ in (49) appropriately. We prove

Lemma 6. It is possible to select the sequence $\left\{d_{v}\right\}$ in (49) such that $M \psi\left|\pi_{\varphi}\right| / S_{\varphi}$ exists if and only if

$$
\frac{1}{\pi} \int_{-\infty}^{\infty} \frac{\psi\left|k_{\varphi}(i t)\right|}{1+t^{2}} d t<\infty
$$

In particular $S_{\varphi} \notin \Theta_{\psi}$ when $\varphi$ is superscalar and $\psi \prec \varphi$, when $\psi$ is subscalar and $\psi \prec \varphi$ or when $\psi \prec \prec \varphi$.

The particular part follows immediately from the fact that we established the crucial property (55) in these cases in (48). The necessity of condition (55) follows immediately from the Szegö-Solomentsev theorem and the representation of a positive, quasi-bounded harmonic function on $H$ which has continuous boundary values.

The sufficiency of condition (55) follows from Heins' methods. First by its construction $\left|k_{\varphi}(i t)\right|$ is increasing with $t(0 \leq t<\infty)$. Consequently $h(t)=\psi\left|k_{\varphi}(i t)\right|$ is increasing. If $h(t)$ is increasing and $\int_{0}^{\infty} \frac{h(t)}{1+t^{2}} d t<\infty$ then the well-known consequence of the inequality

$$
\frac{x h(x)}{\left(1+(2 x)^{2}\right.} \leq \int_{x}^{2 x} \frac{h(t)}{1+t^{2}} d t<\varepsilon
$$

is that $h(t)=o(t)$. Hence

$$
\psi_{i}^{\prime} k_{f}(i t)^{\prime}=o(t) \text {. }
$$

Write $P * \psi\left|k_{\varphi}\right|$ as the convolution of the Poisson kernel with the continuous boundary values of $\psi\left|k_{\varphi}\right|$. Its existence is guaranteed by the crucial property (55). Then $\psi\left|k_{\varphi}\right|-P * \psi\left|k_{\varphi}\right|=u$ is subnarmonic in $H$ with zero boundary values. But $P * \psi\left|k_{\varphi}\right|$ is positive and by $(56) \quad \psi\left|k_{q}(w)\right|=$ $o(|w|)$. Hence

$$
\lim \inf _{r} \max _{\theta} \frac{u\left(r e^{\Theta}\right)}{r} \leq 0
$$

and $M \psi\left|k_{\nu}\right| / H$ exists by the Phragmén-Lindelöf principle. 
Next we consider the truncated surface $S_{p}^{n}$ (see section 4c) and show that $M \psi|\pi| / S_{\varphi}^{n}$ exists. This follows by Heins' argument [1, III 4, p. 46]. For completeness we outline it in this context. First $M \psi|\pi| / \bigcup_{\nu=1}^{N} A_{\nu}$ and $M \psi|\pi| /\left(S_{\varphi}^{n}-\bigcup_{\nu=1}^{N} A_{\nu}\right)$ clearly exist. Let $\Delta_{\nu} \quad(1 \leq v \leq N)$ be copies of closed disks in $A_{v}$ for which $I_{\nu} \subset$ int $\Delta_{\nu} \subset \Delta_{v} \subset$ int $A_{\nu}$ and $\pi\left(\Delta_{v}\right)$ is a disk. Iet $\Delta=\mathrm{U} \Delta_{v}, C=\mathrm{U} \partial \Delta_{v}$ and consider the disjoint union

$$
S_{\varphi}^{n}-C=\Omega_{1} \cup \Omega_{2}
$$

where $\Omega_{2}=\bigcup_{\nu=1}^{N}\left(A_{\nu}-\Delta_{\nu}\right)$ (disjoint union) is the unbounded part and $\Omega_{1}$ is the bounded part. Certainly $M \psi|\pi| / \Omega_{j}$ exists for $j=1,2$. Let $u, v$ have the following properties:

$$
\begin{aligned}
& u, v \text { continuous on } S_{\varphi}^{n}, \\
& u, v \text { harmonic on } \Omega_{1} \cup \Omega_{2}, \\
& u=0 \text { on } \Omega_{1}, v=1 \text { on } \Omega_{1}, \\
& u \leq M \psi|\pi| /\left(\Omega_{1} \cup \Omega_{2}\right), \quad v \geq 0, \\
& u \text { maximal, } v \text { minimal. }
\end{aligned}
$$

Then $u+\psi(n) v$ is a superharmonic majorant of $\psi(\pi)$ on $S_{\varphi}^{n}$ and hence $M \psi|\pi| / S_{\varphi}^{n}$ exists.

Now let $h_{n}=M \psi|\tau| / S_{\varphi}^{n}$. Since $S_{\varphi}^{n} \subset S_{\varphi}^{n+1}$ we have $h_{n} \leq h_{n+1}$ on $S_{\varphi}^{k}(k \leq n)$. Moreover $U S_{\varphi}^{n}=S_{\varphi}$. Evidently if $h=M \psi|\pi| S_{\varphi}$ exists then $h \geq h_{n}$ for all $n$. Conversely if $\lim h_{n}$ exists (finite) then it is harmonic and majorizes $\psi \cdot \pi$. In other words it follows from Harnack's principle that $\lim h_{n}=M \psi / \pi / S_{\text {w }}$ if and only if

$$
h_{n}\left(p_{0}\right) \leq B \quad(n \geq 3)
$$

for some fixed $p_{0} \in S_{\varphi}$. But repeating an argument of Heins similar to the one above $[1$, p. 41] we find that

$$
h_{n+1}\left(p_{0}\right) \searrow h_{n}\left(p_{0}\right) \text { as } d_{n+1} \searrow c_{n+1} \text {. }
$$

Hence if $d_{n+1}$ is selected so that $h_{n+1}\left(p_{0}\right) \leq h_{n}\left(p_{0}\right)+2^{-n}$ the existence of $M \psi|\pi| / S_{\varphi}$ will follow.

c. It is now clear how the main theorem $1(10),(11)$ follows from lemmas 5 and 6.

6. Unions and Intersections. a. If $\varphi$ is finite and subscalar then the strong covering property (47) and the Myrberg representation (54) is valid for $f \in H\left(T_{\varphi}^{3}\right)$ and hence by the proof of lemma 5 we have 


$$
T_{\varphi}^{3} \in \mathscr{C}_{\varphi} .
$$

In addition $M \psi|\pi|$ exists whenever $\psi \prec \varphi$, because the construction of $T_{\varphi}^{3}$ is independent of $\psi$ and the last selection of $\left\{d_{\nu}\right\}$ is not necessary. Hence $\pi \in H_{\varphi}\left(S_{\varphi}\right)$ implies

$$
T_{\varphi}^{3} \notin \Theta_{\psi}
$$

b. Next to show the countable union property (12) we need only select the $\left\{d_{\nu}\right\}$ in a careful manner. First select $\left\{d_{\nu}\right\}_{\nu \geq 1}$ so that $M \psi_{1}|\pi| / S_{\varphi}$ exists. Then set $d_{\nu 3}=d_{\nu}$ and write $S_{\varphi}=S_{\varphi 3}$ for this selection. Next choose $d_{\nu_{4}}$ for $v \geq 2$ such that on this surface $S_{\psi^{4}}$

$$
\begin{gathered}
c_{v}<d_{\nu 4} \leq d_{\nu 3}, \quad(v \geq 2), \\
M \psi_{4}|\pi| / S_{q 4} \text { exists. }
\end{gathered}
$$

Because of (58) we find that $M \psi_{3}|\pi| / S_{q_{4}}$ also exists and

$$
S_{\varphi 3}^{3}=S_{\varphi 4}^{3} .
$$

In general then

$$
\begin{array}{ll}
d_{v k+1}=d_{v k}, & \text { for } v \leq k, \\
c_{\nu}<d_{v k+1} \leq d_{v k}, & \text { for } v \geq k+1, \\
M \psi_{j}|\pi| S_{\varphi k+1} & \text { exists for } 3 \leq j \leq k+1 .
\end{array}
$$

The diagonal sequence $d_{v v}$ gives a surface $S_{\varphi}$ on which

$$
M \psi_{j}|\pi| / S_{\varphi} \quad \text { exists for } j \geq 3 .
$$

c. To obtain the countable intersections result (12) we follow a variation of Heins' construction. Let $S_{\varphi m}$ be constructed such that

$$
S_{\varphi m} \in \mathscr{C}_{q m}-\mathscr{\Theta}_{r} \text {. }
$$

Define the marked copies by

$$
\begin{gathered}
S_{m}=\left\{(p, m): p \in S_{\Upsilon_{m}}\right\}, \\
\pi(p, m)=\tau(p) .
\end{gathered}
$$

Make new cuts by letting $-3+2^{-2 v}=e_{\nu}, \quad-3+2^{-v 2+1}=f_{v}$ and setting

$$
L_{v m}=\left\{(t, 2, m): e_{v} \leq t \leq f_{v}\right\}
$$

for $v, m \geq 1$. Then consider the pieces

$$
\begin{array}{ll}
R_{1}=S_{1}-\bigcup_{\nu=2}^{\infty} L_{\nu 1}, & \text { if } m=1 \\
R_{m}=S_{m}-L_{m m}, & \text { if } m>1,
\end{array}
$$


and weld them together by the criss-cross technique (51). Let $R$ denote the new surface. Clearly the same argument under (54) applies here and hence the Myrberg representation (54) holds on $\mathbf{H}_{\chi}(R)$ for any Orlicz function $\chi$. Using the sectors $A_{v m}$ of $S_{m}$ we see that the proof of lemma 5 implies

$$
R \in \bigcup_{m=1}^{\infty} \mathscr{C}_{\varphi m} .
$$

To see that $M \varphi|\pi| / R$ exists we repeat the truncation-majorization-exhaustion argument of Heins described in detail in section $5 \mathrm{~b}$, to see that (57) holds for

$$
h_{m}=M \varphi|\pi| /\left(R-\bigcup_{\nu=m+1}^{\infty} S_{m}\right)
$$

provided the $\left\{d_{v}\right\}$ and $\left\{f_{v}\right\}$ are appropriately selected (use both diagonals).

7. Questions. The function $\varphi(x) \equiv e^{x}$ is an example of an Orlicz function which is not superscalar. It would appear somewhat doubtful that an infinite Orlicz function could be superscalar.

Second, there is the open question of what relation between $\varphi$ and $\psi$ is necessary and sufficient for $\mathscr{\Theta}_{\varphi} \subset \mathscr{\Theta}_{\psi}$. Does $\Theta_{\varphi} \subset \mathscr{\Theta}_{\psi}$ imply $\varphi \prec \psi$ or does it imply $\varphi \leq K \psi$ and $\varphi\left(r_{n}\right) \leq(1 / n) \psi\left(r_{n}\right)$ for $r_{n} \nearrow \infty$ ? We do not even know whether it implies $\varphi \leq K \psi$. Similar and related questions are open for $\mathscr{C}_{\varphi}=\mathscr{\Theta}_{\psi}$ and $\mathscr{C}_{\varphi} \| \mathscr{\Theta}_{\psi}$.

Third, there are the obvious questions concerning unions and intersections. With new functions $\psi$ (other than Orlicz functions) the question of characterizing " $\subset^{\prime \prime}, "="$, "||" are more complicated. Suppose that the growth condition for which "禹 $\supset \mathscr{\Theta}_{\psi}$ if and only if $\varphi-\psi$ " were solved. We would say that the classification was complete if

$$
\bigcup_{\psi \subset \varphi} \mathscr{Q}_{\psi}=\mathscr{C}_{\varphi}=\bigcap_{\varphi \subset \psi} \mathscr{C}_{\psi} .
$$

Is there a complete classification of surfaces by some collection of null Hardy- $\varphi$ classes? 


\section{Bibliography}

[1] Heins, Maurice: Hardy classes on Riemann surfaces, Forschungsinstitut für Mathematik-series, Springer-Verlag, Berlin-Heidelberg-New York, 1969.

[2] Jenkins, James A. and Oikawa, Koturo: On Results of Ahlfors and Hayman, realling and insert Ill. J. Math., 15 (1971), pp. 664-671.

[3] Warschawski, S. E.: On Conformal Mapping of Infinite Strips, Trans. Amer. Math. Soc., 51 (1942), pp. 280-335.

Case Western Reserve University

Cleveland, Ohio 44106 